\title{
Neurosarcoidosis: Case Report and Brief Literature Review
}

\author{
Andrew V. Pasternak IV MD, MS, and Frank M. Graziano, MD, PhD
}

A 31-year-old African-American man complained of a 1-month history of a new-onset headache. His headache was constant, most intense behind his eyes, and 10 of 10 in severity. He noticed the headache upon awakening, and it persisted throughout the day. Mild photophobia and lightheadedness were associated with the headache. The patient denied visual changes, hearing changes, nausea, vomiting, vertigo, shortness of breath, chest pain, cough, fevers, or chills.

His medical history was remarkable only for hypertension, and he was not taking any medications. He smoked 1 pack of cigarettes per day, used alcohol occasionally, and used no other drugs. The patient had lived in Oklahoma, Texas, Arkansas, and Illinois, and had traveled to Mexico within the past 5 years. His work history included laboring on cargo boats, clipping chicken wings on a chicken farm, and most recently, installing fuel pumps at gas stations. Pertinent physical findings included a blood pressure of $160 / 105 \mathrm{~mm} \mathrm{Hg}$. His pupils were equal and reactive to light, he had full visual fields, and there was no evidence of papilledema or uveitis on an ophthalmologic examination. He also had bilateral strength reduction (4/5) in the proximal lower extremities, but had normal sensation in his lower extremities. Findings of the remainder of his physical examination were unremarkable.

Because of the duration and severity of the headaches, a computed tomographic (CT) scan was ordered. The scan showed an enhancing soft-tissue mass in the posterior left temporal and occipital lobe (Figure 1). The patient was given dexamethasone and mannitol to reduce intracranial pressure. A cerebral angiogram, ordered to establish further the nature of the lesion, showed the lesion to be avascular. A brain biopsy determined that the lesion

Submitted, revised, 22 September 1998. From the Department of Family Medicine, (AVP), University of Wisconsin-Madison, and the Department of Internal Medicine (FMG), University of Wisconsin. Address reprint requests to Andrew V. Pasternak, MD, MS, Washoe Family Care, 975 Ryland Ave, Suite 100, Reno, NV 89502. was a noncaseating granuloma. Fungal, bacterial, and tuberculosis stains of the tissue were all negative. Skin testing for candida and mumps was positive, but a test for tuberculosis (PPD) was negative. Results of a chest radiograph, a chest CT scan, and pulmonary function tests done after the biopsy were unremarkable. The patient's complete blood count and serum protein and calcium levels were normal, and a test for human immunodeficiency virus and a VDRL test were negative. His serum angiotensin converting enzyme (SACE) level was elevated at $95 \mathrm{U} / \mathrm{L}$ (normal 8 to $52 \mathrm{U} / \mathrm{L}$ ). Findings from a full ophthalmologic examination, including slit-lamp examination and dilated fundoscopy, were normal.

A presumptive diagnosis of neurosarcoidosis was made, and the patient was given oral prednisone at $60 \mathrm{mg} / \mathrm{d}$. Triple antituberculosis therapy and amphotericin B were also prescribed. When all cultures and serologic reports came back negative, these drugs were stopped. The patient's hypertension was controlled by sustained-release nifedipine.

With corticosteroid therapy the patient's headaches dramatically improved, and his SACE level decreased to $65 \mathrm{U} / \mathrm{L}$. During the next several months, the patient did well. Tapering the prednisone dose, however, led to recurrence of the headaches along with blurred vision and bilateral lower extremity numbness. On a lumbar puncture, his opening pressure was elevated $(45 \mathrm{~mm}$ of water), and his cerebral spinal fluid (CSF) glucose level was $108 \mathrm{mg} / \mathrm{dL}$ and CSF protein level was 70 $\mathrm{mg} / \mathrm{dL}$. Magnetic resonance imaging (MRI) showed the same temporo-occipital mass with new enhancement along the interhemispheric fissure above the corpus callosum. The patient's prednisone was increased to $60 \mathrm{mg} / \mathrm{d}$, and oral methotrexate was added as a potential corticosteroidsparing agent. The patient's symptoms again improved with this therapy.

Approximately 2 months later, again while tapering his corticosteroid therapy, the patient developed mental status changes, memory problems, and worsening bilateral lower extremity weakness. 


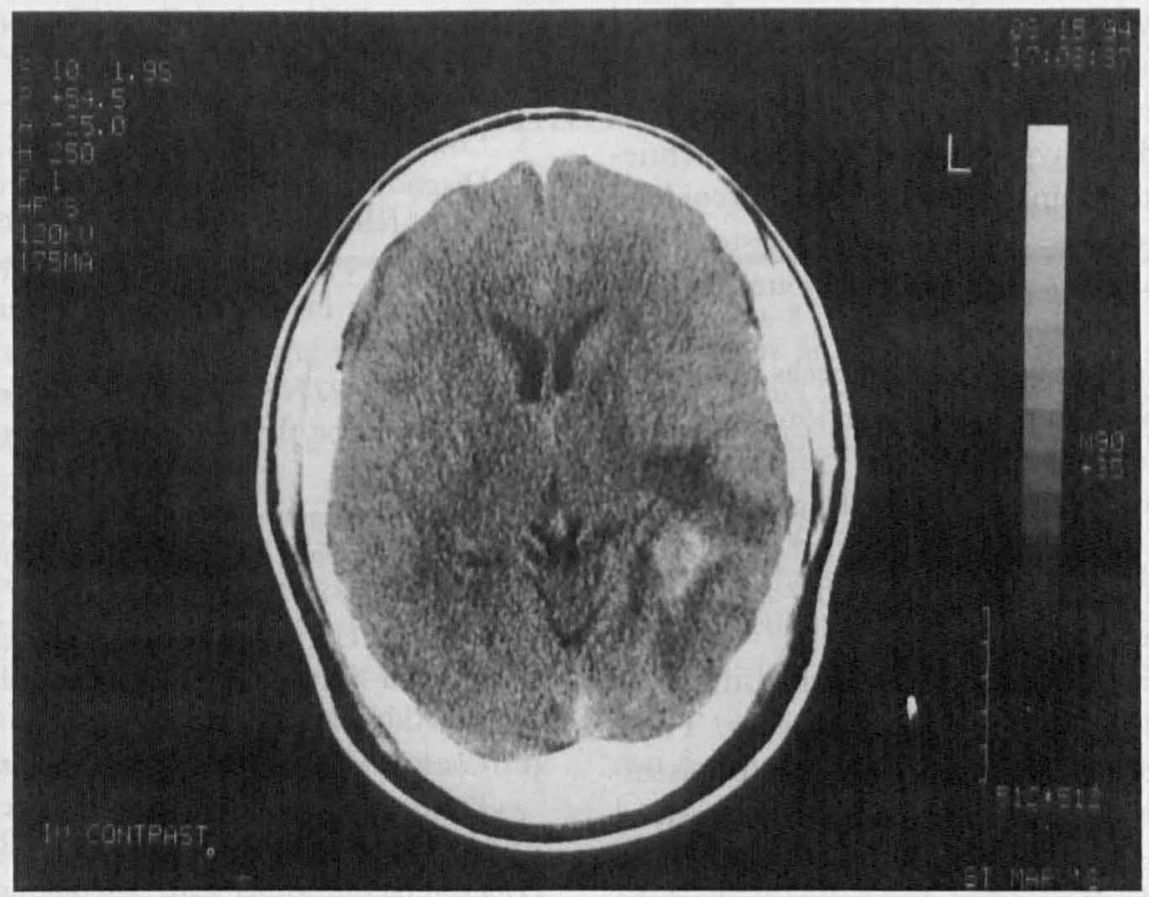

Figure 1. Conputed tomographic scan showing soft tissue mass in posterior left temporal and occipital lobe.

While the SACE had normalized (47 U/L) and a head MRI showed a decrease in the left temporal lobe lesion, an MRI of the cervical and thoracic spine showed patchy lesions consistent with sarcoid. An electroencephalogram showed sharp and low-wave complexes in the left temporal posterior and left frontal regions believed to be epileptiform. As a result, phenytoin was prescribed (Dilantin), the prednisone was again increased to $60 \mathrm{mg} / \mathrm{d}$, and methotrexate was discontinued.

Since the diagnosis 3 years ago, we have been unable to taper the patient's prednisone dose below $20 \mathrm{mg} / \mathrm{d}$ because of the resulting recurrent headaches, bilateral lower extremity weakness, visual changes, and behavior changes. Azathioprine and hydroxychloroquine (Plaquenil) have been added to the patient's drug regimen but have not been completely successful as corticosteroid-sparing agents. His most recent head MRI, however, showed no evidence of a temporal lobe lesion and no lesions in the cervical or thoracic spine.

\section{Literature Review}

Sarcoidosis is a systemic disease most commonly affecting the lungs and lymphatic system. Sarcoidosis has a higher incidence in African-Americans and usually appears in the third or fourth decade of life. Biopsies of sarcoid lesions typically show histologic findings of noncaseating granulomas. Other causes for noncaseating granulomas that should be considered include berylliosis, syphilis, Crohn disease, primary biliary cirrhosis, fungal infections, and mycobacterial infections. ${ }^{1}$ Sarcoidosis remains a diagnosis of exclusion after appropriate evaluation for the other diseases that can cause granulomatous disease. If a clinician suspects sarcoidosis, patients should be examined carefully for lesions in the lymph nodes, liver, spleen, eyes, heart, parotid gland, and thyroid. In patients with sarcoidosis, $16 \%$ to $25 \%$ will have skin involvement including erythema nodosum, lupus pernio, and assorted plaques, nodules, papules, and macules. ${ }^{1,2}$ Depending on the location of lesions, skin biopsy and transbronchial lung biopsies have the best yield and lowest morbidity for diagnosis. ${ }^{3} \mathrm{~A}$ brain biopsy was performed with this patient because of concern about a primary tumor of the central nervous system.

Approximately $5 \%$ to $10 \%$ of patients with sarcoidosis develop neurosarcoidosis. ${ }^{4,5,6}$ The racial distribution of neurosarcoidosis does not differ much from that for sarcoidosis. ${ }^{6,7}$ Some studies have shown patients with neurosarcoidosis are slightly younger than the expected age distribution for sarcoidosis, but other studies have found no age difference between patients with sarcoidosis and neurosarcoidosis. ${ }^{5,8}$

Most patients with neurosarcoidosis have only minimal symptoms. The most common sign is cra- 
nial nerve involvement including seventh facial nerve palsy. Patients can also complain of headaches or seizures. Less common signs include intracranial bleeding, hypertension, hyperprolactine$\mathrm{mia}$, and diabetes insipidus. Rarely does sarcoidosis occur as a mass in the central nervous system, and in most cases patients are ultimately found to have sarcoid lesions elsewhere in the body. ${ }^{9-12}$ Our patient was somewhat unique. Whereas his brain mass was pathologically a sarcoid lesion, he had no evidence of sarcoidosis outside the central nervous system based on a chest radiograph, gallium scan, and chest CT. Isolated sarcoidosis of the central nervous system has been reported only a few times in the literature. ${ }^{13,14}$

The diagnosis of neurosarcoidosis has improved with the advent of CT and MRI imaging. These imaging procedures have allowed for better visualization of lesions in the central nervous system. SACE levels are not helpful in the diagnosis of neurosarcoidosis. In one series, only one half of the patients with neurosarcoidosis had elevated SACE levels. ${ }^{5}$ Prognosis for patients with neurosarcoidosis depends on the extent of involvement. Patients with limited facial nerve involvement usually have a good outcome. Patients with cerebral or spinal masses, hydrocephalus, or seizures tend to have a worse prognosis. ${ }^{15,16}$

Corticosteroids are the cornerstone of treatment for neurosarcoidosis. Early studies have shown good results with corticosteroids alone, but recent studies have shown patients have better outcomes when adjuvant therapies are added. ${ }^{3}$ When corticosteroids alone fail to control symptoms and shrink lesions, other options for treatment include azathioprine, ${ }^{17}$ methotrexate, ${ }^{18}$ cyclophosphamide, ${ }^{19}$ hydroxychloroquine, ${ }^{5}$ and radiotherapy. ${ }^{20}$ Cyclosporin has also been used with mixed results. ${ }^{21,22}$ Most of these studies, however, are either anecdotal reports or have few patients in the study. In this patient temporal lobe mass and spinal lesions disappeared with therapy, but he continues to have clinical symptoms despite adjuvant therapies.

\section{References}

1. Newman LS, Rose CS, Maier LA. Sarcoidosis. N Engl J Med 1997;336:1224-34.

2. Peckham DG, Spiteri MA. Sarcoidosis. Postgrad Med J 1996;72:196-200.

3. Gilman MJ, Wang KP. Transbronchial lung biopsy in sarcoidosis. An approach to determine the optimal number of biopsies. Am Rev Respir Dis 1980;122: 721-4.
4. Delaney P. Neurologic manifestations in sarcoidosis: review of the literature with a report of 23 cases. Ann Intern Med. 1977;87:336-345.

5. Lower EE, Broderick JP, Brott TG, Baughman RP. Diagnosis and management of neurological sarcoidosis. Arch Intern Med 1997;157:1864-8.

6. Oksanen V. Neurosarcoidosis: clinical presentations and course in 50 patients. Acta Neurol Scand 1986; 73:283-90.

7. Sharma OP. Neurosarcoidosis: a personal perspective based on the study of 37 patients. Chest 1997; 112:220-8.

8. Stern BJ, Krumholz A, Johns C, Scott P, Nissim J. Sarcoidosis and its neurological manifestations. Arch Neurol 1985;42:909-17.

9. Veres L, Utz JP, Houser OW. Sarcoidosis presenting as a central nervous system mass lesion. Chest 1997;111:518-21

10. Clark WC, Acker JD, Dohan FC Jr, Robertson JH. Presentation of central nervous system sarcoidosis as intracranial tumors. J Neurosurg 1985;63:851-6.

11. Powers WJ, Miller EM. Sarcoidosis mimicking glioma: case report and review of intracranial sarcoid mass lesions. Neurology 1981;31:907-10.

12. Cahill DW, Botero E, Robinson WL. Neurosarcoidosis presenting as an intracranial mass. J Fla Med Assoc 1986;73:35-9.

13. Cariski AT. Isolated CNS sarcoidosis. JAMA 1981; 245:62-3.

14. Sommer N, Weller M, Petersen D, Wietholter $H$, Dichgans J. Neurosarcoidosis without systemic sarcoidosis. Eur Arch Psychiatry Clin Neurosci 1991; 240:334-8.

15. Luke RA, Stern BJ, Krumholz A, Johns CJ. Neurosarcoidosis: the long-term clinical course. Neurology 1987;37:461-3.

16. Krumholz A, Stern BJ, Stern EG. Clinical implications of seizures in neurosarcoidosis. Arch Neurol 1991;48:842-4.

17. Agbogu BN, Stern BJ, Sewell C, Yang G. Therapeutic considerations in patients with refractory neurosarcoidosis. Arch Neurol 1995;52:875-9.

18. Lower EE, Baughman RP. Prolonged use of methotrexate for sarcoidosis. Arch Intern Med 1995;155; 846-51.

19. Demeter SL. Myocardial sarcoidosis unresponsive to steroids: treatment with cyclophosphamide. Chest 1988;94:202-3.

20. Bejar JM, Kerby GR, Ziegler DK, Festoff BW. Treatment of central nervous system sarcoidosis with radiotherapy. Ann Neurol 1985;18:258-60.

21. Kavanaugh AF, Andrew SL, Cooper B, Lawrence EC, Huston DP. Cyclosporin therapy of central nervous system sarcoidosis. Am J Med 1987;82:387.

22. Cunnah D, Chew S, Wass J. Cyclosporin for central nervous system sarcoidosis. Am J Med 1988;85:580-1. 